\title{
The Influence of Concave Pectoral Fin Morphology in The Performance of Labriform Swimming Robot
}

\author{
Farah Abbas Naser*1, Mofeed Turky Rashid ${ }^{2}$ \\ ${ }^{1}$ Electrical Engineering Department, University of Basrah, Basrah, Iraq \\ ${ }^{2}$ Electrical Engineering Department, University of Basrah, Basrah, Iraq
}

Correspondence

* Farah Abbas Naser

Electrical Engineering Department,

University of Basrah, Basrah, Iraq

Email: farahabbasnaser@gmail.com

\begin{abstract}
Swimming performance underlies the biomechanical properties and functional morphology of fish fins. In this article, a pair of concave fin has been suggested, which is inspired from Labriform-mode Swimming fish. First, three concave fins with different sizes are proposed in order to choose the optimum size. All three fins have the same length but with different surface areas, such that each fin has an aspect ratio different from the others. Next, the complete design of the robot is suggested, the complete design of the body and pectoral fins were subjected to computational fluid dynamics (CFD) analysis to show the validity of the proposed model. Finally, the physical model is suggested and provided with $3 D$ printer of Polylactic Acid (PLA) with a density of $1240 \mathrm{~kg} / \mathrm{m}^{3}$. The swimming robot fins have been examined by CFD analysis provided by Solidworks ${ }^{\circledR}$ to evaluate the highest thrust and lowest drag forces. The result showed that the optimum fin is the one with the lowest aspect ratio fin produces the highest drag, whereas the highest aspect ratio fin gives the lowest drag and thrust, therefore; a value of aspect ratio in between these two cases is chosen. While other types of examinations are based on motion analysis of the 3D design, the required motor torque is calculated in order to select a suitable servomotor for this purpose, which a HS-5086WP waterproof servomotor can achieve the calculated torque.
\end{abstract}

KEYWORDS: Pectoral Fin, Labriform Mode, Concave Fin, Swimming Robot, Computational Fluid Dynamics (CFD).

\section{INTRODUCTION}

In recent decades, the bionic propulsion systems that employ mechanisms obtained from fish swimming have been highly utilized in the propulsion of underwater robots [1-4]. Two main swimming categories of fish are typically named after the body and/or caudal fin (BCF) swimming modes and median and/or paired fin (MPF) swimming modes [5-6]. According to the type of fins that are used in swimming locomotion, they can be further classified into the undulatory type and oscillatory type [7].

In the BCF-undulatory type, the motion generated in this motion is like a wave form generated from fish head to its tail such as, Carangiform, and Subcarangiform mode. In the oscillation type, the motion is generated by turning the body or caudal fin to propel the fish such as Ostraciiform and Thunniform mode. The other mode of motion swimming is the MPF-undulatory gymnotiform, Amiform, and Balistiform locomotion where oscillatory-type of motion involves Labriform and Tetadntiform. Whereas Another type of MPF that depends both on undulatory and oscillatory locomotion is known as Rajiform [8-9]. About $15 \%$ of the fish swims in non-BCF modes as the main propulsion mechanism, while there are very large number are basically based on BCF modes for propulsion which MPF modes for stabilization and maneuvering purposes [10].

In Labriform mode, propulsion is achieved by MPFoscillatory movements of the pectoral fins [11]. Two types were identified in Labriform mode: a "rowing " (drag-based) and a "flapping" (lift-based) in [12]. According to [13], at low speeds, drag based are more efficient when the flow of chordwise fin is small. While they are more efficient at higher speeds in lift-based motion.

There are many different kinds of fish fins. Each fin of a fish helps in swimming and maneuvering. For each fish, generally, there are five main fins follows: Dorsal, Pelvic, Caudal (tail), Anal and Pectoral as shown in Fig. 1. Dorsal fins are located either on the back of the fish or its top, it helps the fish during sharp turning or stops. Fish may have up to three different kinds of dorsal fins, known as proximal, middle, and distal dorsal fins, however, many fish have just two dorsal fins with the middle and distal fins merged together. Dorsal fins types are: Single, Split, Pointed, Trigger, Spine Triangular and Trailing. In the other hand, T caudal fin or commonly known a tail fin, can be considered 
as the primary fin that gives the forward thrust and speed up fish velocity in many kinds of fish. In bony fist there are many different kinds of caudal fins as: Indented, Round, Square, Forked, Lunate, and Pointed. The pair of ventral or pelvic fins, is located on the bottom front of the fish which helps in fish stability and slow down the fish velocity also it is useful during sudden stopping. The Anal fin supports the dorsal fins by making it more stable in the water.

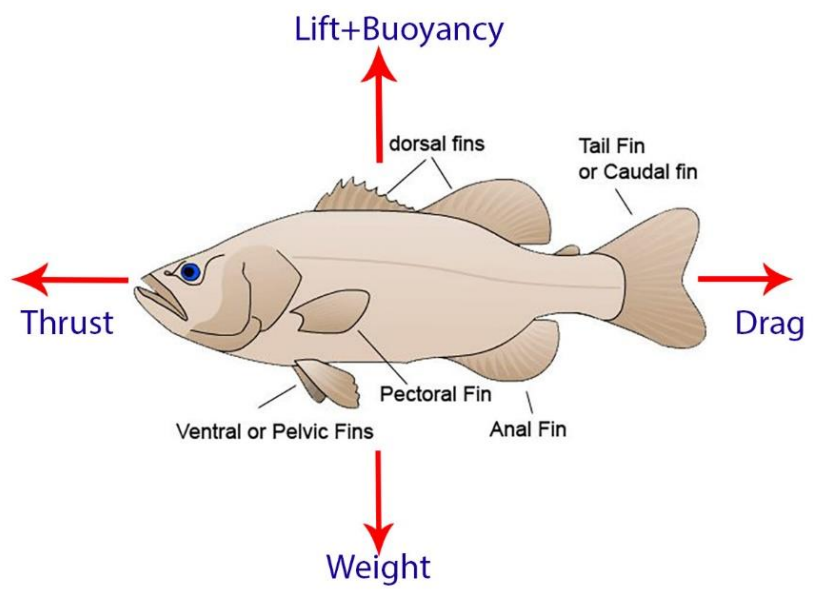

Fig. 1: Different types of fish fins.

Pectoral Fin shape has important effects for swimming hydrodynamics, usual habitat uses and energetics as shown in [14]. Many different variables that describe fin shape, one of them is the aspect ratio (Ar), which can be defined as the measure of the relative narrowness of the fin, in labriform swimming fish, for example, pectoral fin shape varies from low $\operatorname{Ar}$ (i.e. $\leqslant 2.0)$ as in paddle-shaped fins, to relatively high $\operatorname{Ar}($ i.e. $\geqslant 4.5)$ [15-16].

In recent pieces of research show that labriform based fish with a lower Ar of paddle-shaped, swims more slowly and occupy a less energetics areas on the reef [17-18] compared to labriform-based with higher Ar of wing-shaped fins. Practical studies have confirmed casual observations that labriform-based with wing-shaped fins use a flapping mode while labriform-based with paddle-shaped fins use a rowing or intermediate mode [16]. A composition of these two studies of flapping and rowing gives support for the rowing-flapping model study. Finally, it is worthy to observe that fish that swims with pectoral fin flapping can achieve speeds higher than that of BCF swimmers of the same size, while the rowing pectoral fins have lower than performance than expected [14]. Labriform mode with pectoral fin motions with steady swimming concluded as Hydrolagus [19], Gomphosus [14,20], Cirrhilabrus [14], Tautoga, Scarus [21], and Lactoria, Tetrasomus [22] [33].

The objective of the present study is to investigate the efficient production of thrust during the rowing motion of Labriform mode, in which the thrust is provided by pectoral fins only. Three concave fins have different sizes is proposed, in order to choose the optimum one, the fins have been experimentally validated by computational fluid dynamics (CFD) analysis provided by Solidworks ${ }^{\circledR}$ to show the most proper one that produces the highest thrust and lowest drag forces, all the three fins have the same length but with different surface areas, such that each fin has an aspect ratio different from the others. It is well known that the lowest aspect ratio fin will produce the maximum thrust in case of BCF mode, but in our case, MPF has been chosen with a concave design, in which the results dramatically changed. The lowest aspect ratio fin (fin 1 in section 2) produces the highest drag, whereas the highest aspect ratio fin (fin 3 in section 2) gives the lowest drag and thrust, however an intermediate aspect ratio fin (fin 2 in section 2) will produce a valuable thrust and an accepted drag. Next the complete design of the robot is suggested, the complete design of the body and pectoral fins were subjected to CFD analysis to show the validity of the proposed model.

After classified the various fin types in the introduction, the next section presents the suggested concave shape of our swimming robot, covering the general characteristics of the hydrodynamic forces acting on a swimming robot as well as kinematic data and mathematical models. Section 3 presents the simulation results of the proposed model and finally, section 4 concludes with some discussion on the relevance to underwater robot design.

\section{Description of The Proposed Design}

Incompressibility and density are the main properties of water as a locomotion environment, these two properties play a significant effect fish evolution. The water is considered as an incompressible fluid, therefore; any movement occurred by an aquatic animal will let the water surrounding it in motion and vice versa. The density of the water is approximately 800 times larger than of air. That is great kinds of swimming proposers had come to light [23-24].

Swimming comprises momentum exchange between the fish and the water that surrounding it. The main momentum exchange occurs via drag, lift and acceleration reaction forces [24]. Drag force swimming consists of the following components:

(1) Skin friction also known as (viscous or friction drag) that occurs between the fish and the boundary layer of water.

(2) Pressures formed also known as (form drag), in pushing water away for the fish to pass.

(3) Induced drag also known as (vortex drag) when the energy lost in the vortices formed by the caudal and pectoral fins while they generate thrust or lift forces.

The last two components are merged in description as pressure drag.

In order to swim in a constant speed, the total forces and momentums acting on a fish should be balanced according to the momentum conservation principle. The main parameters that determine the momentum transfer mechanisms to thrust and resistance are Reynolds number, reduced frequency, and overall shape. The Reynolds number (Re)can be considered as the ratio of inertial forces over viscous forces as given bellow [25]:

$$
\operatorname{Re}=\frac{L U}{\lambda}
$$

where $L$ is a characteristic length (for fish body or fin), $U$ is the swimming velocity and $\lambda$ is the kinematic viscosity of 
water. Generally, Re in adult fish where inertial forces are dominant and often the viscous forces are neglected. Here, Re pressure, acceleration reaction drag and lift mechanisms can all cooperate in generating effective forces.

In the other hand, the reduced frequency $\sigma$ indicates the importance of unsteady (time-dependent) effects in the flow and is defined as:

$$
\sigma=2 \pi \frac{f L}{U}
$$

Where $f$ is the oscillation frequency. The reduced frequency basically makes a comparison between the time taken for a particle to traverse the length of an object and the time taken to complete one cycle of movement. Also it is a measure of relative importance of acceleration reaction to pressure drag and lift forces. When $\sigma<0.1$, it is a steady movement and acceleration reaction forces have little impact. When $0.1<\sigma$ $<0.4$ all three mechanisms of force generation are important, whereas at larger values of $\sigma$, acceleration reaction dominates. practically, the reduced frequency rarely seems below the threshold of 0.1 [24].

Finally, the shape of the swimming fish largely affects the magnitude of the force components. Grate number of studies about the relationship in steady state lift and drag forces generation, in the other hand, little work has been done on the connection between shape and acceleration reaction. One measure of swimming efficiency is Froude number where efficiency $\eta$, defined as:

$$
\eta=\frac{\langle T\rangle U}{\langle P\rangle}
$$

$U$ is the mean forward velocity of the fish, $\langle T\rangle$ is the time-averaged thrust produced and $\langle P\rangle$ is the time-averaged power required [26-27].

Measurement of pectoral fin shape has been studied in terms of $\mathrm{Ar}$ (see Fig. 2). The leading edge squared, divided by the surface area of the spread fin [14] [28] as follows:

$$
A r=\frac{P F_{L}^{2}}{S}
$$

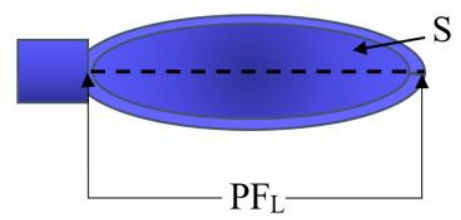

Fig.2: Proposed pectoral fin

Where $P F_{L}$ is the pectoral fin length and $S$ is the projected surface area. By providing an estimate of the roundness or extent of taper in fin shape, these measurements have been directly related to speed performance and thrust generation. Tapered, high Ar fins are usually used by the fish to produce lift-based thrust and maintain high speeds, whereas rounded, low Ar fins are basically associated with rowing fin oscillation of drag-based thrust and effective at low-speed maneuverability.

Another factor that assets on swimming efficiency is Strouhal number (St), for biological fish is usually in the range of $0.05-0.6$ [26-30]. St is related to how fast vortices are being shed into the wake of an oscillating foil and the space between the vortices at each half stroke. St is defined as:

$$
S t=\frac{2 f\left(P F_{L}\right) \sin A}{|U|}
$$

where $\mathrm{A}$ is the peak-to-peak fin oscillating amplitude.

In accordance to the Newton's Second Law, the motion description follows the equation of:

$$
\sum F_{b}=\sum M g
$$

Where $M$ is the total mass vector of the body (including added mass), and $g$ is the gravitational vector force. we assume both the body and the world's frame are coinciding, the body of the robot are neutrally buoyant, only surge and sway components of $\mathrm{x}_{\mathrm{b}}, \mathrm{y}_{\mathrm{b}}$-plane will be considered, other velocity components will be neglected. The robot is assumed to be surrounded by inviscid water it moves in xy- plane, under the assumptions that inertial coupling between the motions of sway, surge, and yaw is neglected; the dynamic equation in a plane of a rigid body is governed by Kirchhoff's equation and written as follows as shown in Fig. 3.

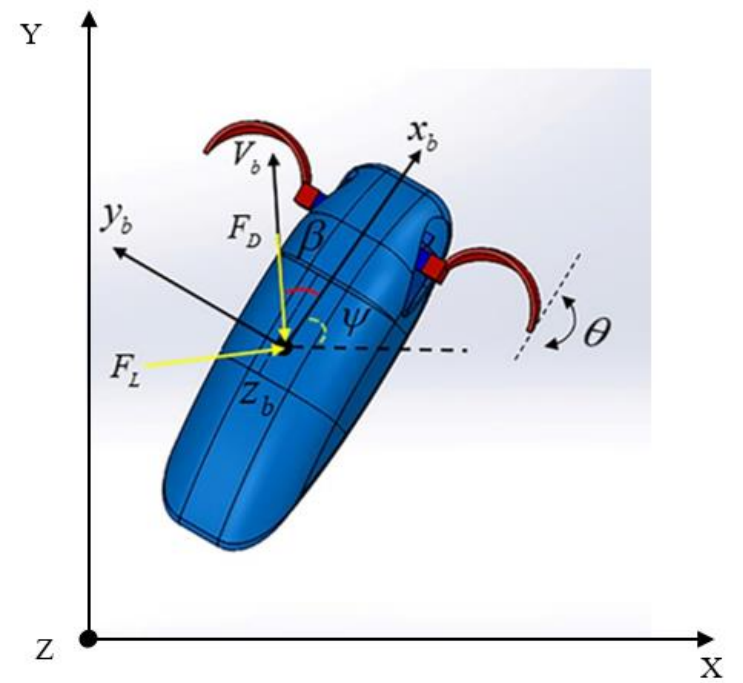

Fig.3: The free body diagram of the swimming body.

$$
\left.\begin{array}{l}
\left(M_{b}-M_{\dot{u}_{a}}\right) \dot{u}=\left(M_{b}-M_{\dot{v}_{a}}\right) v w_{z}+F_{b x} \\
\left(M_{b}-M_{\dot{v}_{a}}\right) \dot{v}=-\left(M_{b}-M_{u_{a}}\right) u w_{z}+F_{b y} \\
\left(I_{z}-N_{\dot{w}_{z}}\right) \dot{w_{z}}=M_{b z}
\end{array}\right\}
$$

Where $M_{b}$ is robotic fish mass, $I_{z}$ is the robot inertia about the $\mathrm{Z}_{\mathrm{b}}$-axis, $M_{u_{a}}, M_{v_{a}}$, and $N_{w_{z}}$ are the added mass/inertia on the rigid body. $u, v$, and $w$ are the body's surge, sway and yaw linear velocity components, respectively.

$$
\left.\begin{array}{l}
F_{b x}=F_{b x_{h}}-F_{D} \cos \beta+F_{L} \sin \beta \\
F_{b y}=F_{b y_{h}}-F_{D} \sin \beta-F_{L} \cos \beta \\
M_{b z}=M_{b z_{h}}+M_{D}
\end{array}\right\}
$$

Where $F_{b x_{h}}, F_{b y_{h}}$ and $M_{b z_{h}}$, and $F_{z}$ are the hydrodynamic forces / moments transmitted from the pectoral fins to the robotic fish. $F_{D}, F_{L}$ and $M_{D}$, are the body's drag, lift and moment forces, respectively. These forces are given as: 


$$
\left.\begin{array}{l}
F_{D}=1 / 2 \rho V_{b x}^{2} S C_{D} \\
F_{L}=1 / 2 \rho V_{b y}^{2} S C_{L} \\
M_{D}=-C_{M w z}{ }^{2} \operatorname{sgn}\left(w_{z}\right)
\end{array}\right\}
$$

Where $\rho$ is the water density, $\mathrm{S}$ is the projected surface area, is the angle between $\mathrm{X}$-axis of global coordinate and $\mathrm{x}_{\mathrm{b}}$ - axis of body coordinate. $C_{D}, C_{L}$ and $C_{M}$ are the drag, lift and moment coefficients, respectively. $\beta$ is defined as the angle of attack of the body, and $\operatorname{sgn}($.$) is the signum function. The$ kinematics of the robot is given as:

$$
\left.\begin{array}{l}
\dot{X}=u \cos \psi-v \sin \psi \\
\dot{Y}=v \cos \psi-u \sin \psi \\
\dot{\psi}=w_{z}
\end{array}\right\}
$$

Where $\psi$ is the angle between the $\mathrm{x}_{\mathrm{b}}$-axis of the body and $\mathrm{X}$-axis of the global reference frame. Generally, the drag force is the force that is in the opposite direction to the flow and the lift force is the normal force to the flow [20-21]. In order to evaluate the hydrodynamic force applied by the water, we set up the proposed model as stationary and let the water be the moving part. The servomotor is employed to a rotating fin based on the following equation:

$$
\theta(t)=A / 2-A / 2 \cos \Omega t
$$

Where $A$ is the oscillation amplitude in degree and $\Omega$ is the angular frequency and can be expressed as $\Omega=2 \pi f$. The added masses, added inertia and wetted surface area were calculated by approximating the robot body as a prolate spheroid accelerating in the fluid [27]. The body inertia was evaluated about $\mathrm{z}$-axis as:

$$
\left.\begin{array}{l}
I_{z}=\frac{1}{5} m_{b}\left(a^{2}+c^{2}\right) \\
a=\frac{\text { Length of the body }}{2} \\
c=\frac{\text { Body width }}{2}
\end{array}\right\}
$$

Where $a$ and $b$ are the lengths of the semi axis of the swimming body,

$$
\left.\begin{array}{l}
M_{\dot{u}_{a}}=-k_{1} m_{f} \\
M_{\dot{v}_{a}}=-k_{2} m_{f} \\
N_{\dot{w}_{a}}=-k^{\prime} I_{f z}
\end{array}\right\}
$$

Where the positive constants $k_{l}, k_{2}$, and $k$ ' are Lamb's $\mathrm{k}$-factors that depend only on the geometry of the body, see [33]. $m_{f}=\rho \forall_{s}$, is the displaced fluid mass, where $\forall_{s}=4 / 3 \pi a b^{2}$ is the volume of the spheroid and $\rho$ is the water density. In (13) $I_{f z}=1 / 5 m_{f}\left(a^{2}+b^{2}\right)$ is the moment of inertia of the fluid's spheroidal mass.

\section{EXPERIMENTS RESULTS}

Three pectoral fins have different surface areas that have been fabricated, these fins are named as Fin1, Fin2 and Fin3. The specifications of these fins are summarized in Table I. Each fin has the same length $(\mathrm{L}=5 \mathrm{~cm})$ but with different revolution angle about its diameter, as shown in table 1, which result in different Ar as shown in Figs. 4 and 5.
TABLE I

RIGID FINS SPECIFICATIONS

\begin{tabular}{|c|c|c|c|c|c|}
\hline $\begin{array}{c}\text { Fin } \\
\text { type }\end{array}$ & $\begin{array}{c}\text { Outer } \\
\text { radius }\end{array}$ & $\begin{array}{c}\text { Inner } \\
\text { radius }\end{array}$ & $\begin{array}{c}\text { Geometrical } \\
\text { revolving angle }\end{array}$ & $\begin{array}{c}\text { Projected } \\
\text { surface area }\end{array}$ & $\begin{array}{c}\text { Mass of } \\
\text { each fin }\end{array}$ \\
\hline Fin1 & $2.5 \mathrm{~cm}$ & $2.3 \mathrm{~cm}$ & $90^{\circ}$ & $16.62 \mathrm{~cm}^{2}$ & $5.00 \mathrm{gm}$ \\
\hline Fin2 & $2.5 \mathrm{~cm}$ & $2.3 \mathrm{~cm}$ & $45^{\circ}$ & $8.31 \mathrm{~cm}^{2}$ & $2.82 \mathrm{gm}$ \\
\hline Fin3 & $2.5 \mathrm{~cm}$ & $2.3 \mathrm{~cm}$ & $22.5^{\circ}$ & $4.15 \mathrm{~cm}^{2}$ & $1.76 \mathrm{gm}$ \\
\hline
\end{tabular}
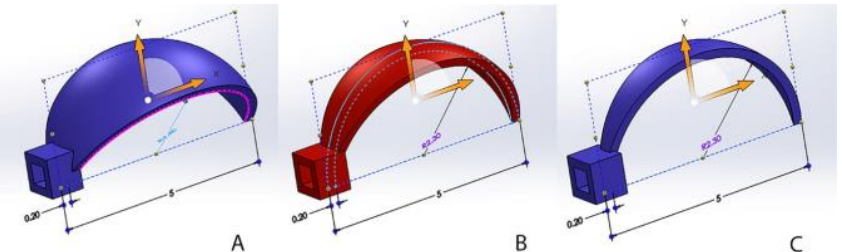

Fig. 4: Three different concave pectoral fins.

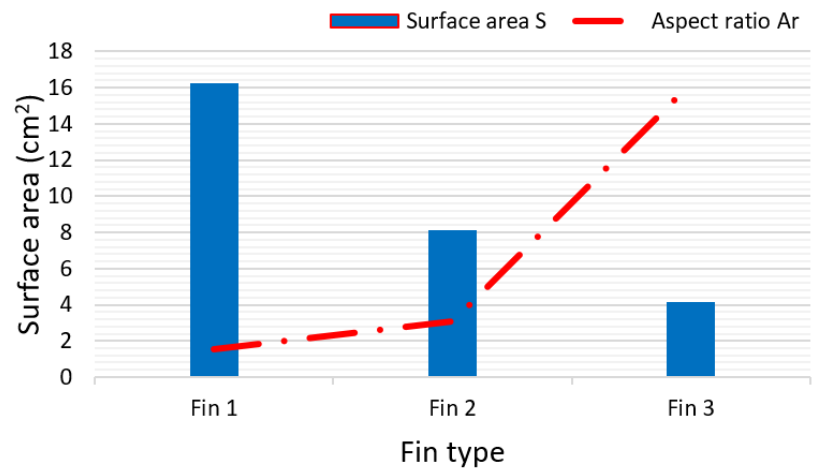

Fig. 5: Aspect ratio of each fin.

These fins have been individually tested by CFD, a computational domain is set to be enough to evaluate each fin as shown in Fig. 6. To show some realistic results, flow type has been set as (laminar and turbulent) with a static pressure of $101325 \mathrm{~Pa}$ at $293.2 \mathrm{~K}$ and a local mesh of six levels of refinement cells are used throughout this simulation.
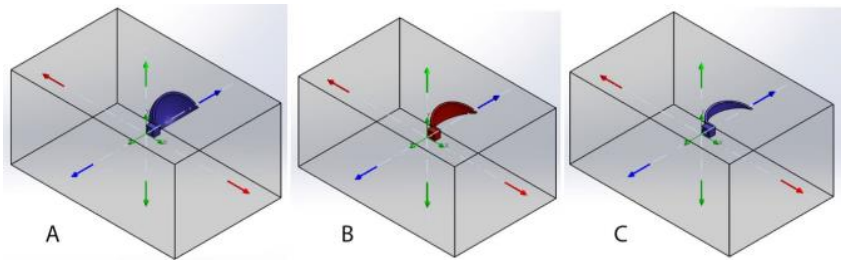

Fig. 6: CFD domain for each of individual fin. A) Fin1. B) Fin2. C) Fin3.

Since rowing motion in Labriform mode is a "drag-based", the generated hydrodynamic forces (thrust) should be at maximum during the power stroke (i.e. when the fin starts to hit) and minimum during recovery stroke (i.e. when the fins returned back to its initial position).

During the power stroke as shown in Fig. 7, Each fin has been subjected to a flow of $0.0145 \mathrm{~m} / \mathrm{s}$ in the $\mathrm{x}$-direction. Flow trajectories provided in Fig. 7, shows that Fin 1 with the lowest aspect ratio Ar has the highest degradation of speed due to its design with larger surface area as expressed in (9), this will be generating the highest drag generation, as a consequence, will affect the total thrust generated. For Fin3 that has the highest aspect ratio Ar, produces the highest drag, but the total generated thrust is not sufficient to accelerate the proposed swimming robot as tested 
experimentally. While an intermediate aspect ratio of Fin2 will produce a valuable thrust and an accepted drag.

This scenario is examined with CFD analysis, with 300 iterations to ensure the stabilization of the final values. These results are summarized in Figs. 8 and 9, where they showed the drag force and drag coefficient respectively.

Next, the 3D model of the proposed swimming robot is implemented, the body is designed with a cross-sectional area approximately as an ellipse form where its size gradually changes through the longitudinal axis of the body in order to reduce water resistance.

The 3D design of the robot is then analyzed with CFD, to ensure that the robot can withstand the surrounding environmental conditions in two cases of power and recovery strokes as shown in Fig. 10 we can see the flow velocity decrease behind the robot as expected, this little degradation in speed will lead to a change in pressure as shown in Fig. 11, however, the maximum reached value of pressure is not so much larger than the already set value of pressure of $101325 \mathrm{~Pa}$ at the beginning of the simulation, this ensures the swimming robot has the ability to stand the external flow changes.

Following our results in [11] and [25] where a variation in input signal of power stroke speed and recovery stroke speed had taken place as shown in Fig. 12, the results showed that the optimum velocity when the power stroke speed is one-third of the recovery stroke speed, the reader can refer to these references for further information, where 1,2,3,4, and 5 represent five signals of power to recovery stroke ratio. The signal is set to complete the power stroke during the first one-third of the time, and the recovery stroke at the last two-thirds of the time. The starting angle of rotation is set to $50^{\circ}$. The robot will rotate in a $100^{\circ}$ amplitude (i.e. from $50^{\circ}$ to $-50^{\circ}$ ). The frequency is set to $1.515 \mathrm{~Hz}$ to match the servo motor specifications.

A computational domain of $(1 \times 0.65 \times 0.65)$ meter in (length, width, and height) has been used as shown in Fig. 13 to match the dimension of the physical swimming pool, which is made of acrylic plastic material. The servomotors of robot pectoral fins are controlled by an Atmega microcontroller. While the torque is calculated at the highest required speed at the power to recovery ratio of $5: 1$, which the maximum value is $0.23 \mathrm{~N} . \mathrm{m}$. This value can be translated to match $3 \mathrm{Kg} / \mathrm{cm}$ where a Hitec 35086W HS-5086WP waterproof digital servomotor has been used as shown in Fig.14. All plastic parts such as the robot body, fins, joints have been printed by a 3D printer of PLA material. The robot motion is captured through Kodak high-resolution camera at a frame rate of 30 frames per second.

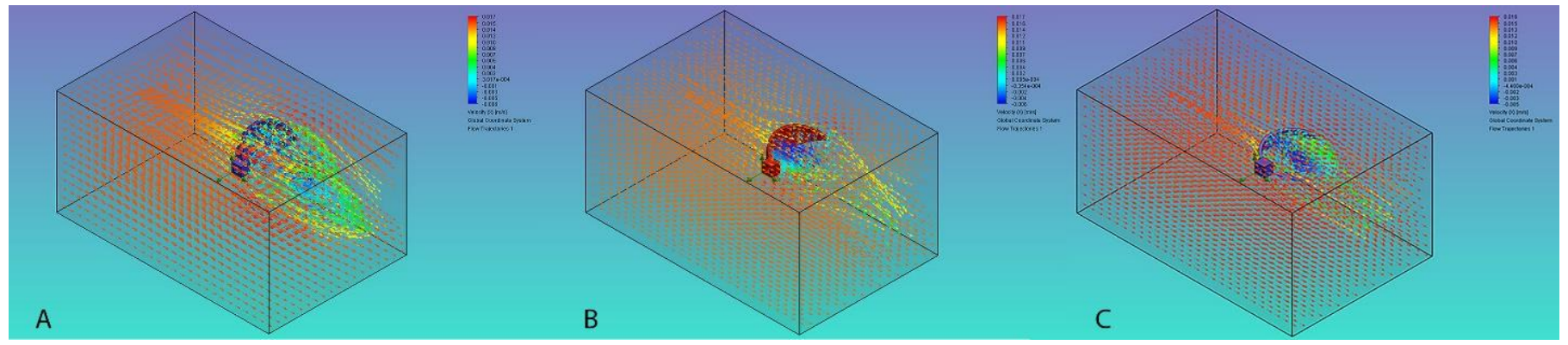

Fig.7: Velocity trajectories during power stroke. A) Fin1. B) Fin2. C) Fin3.

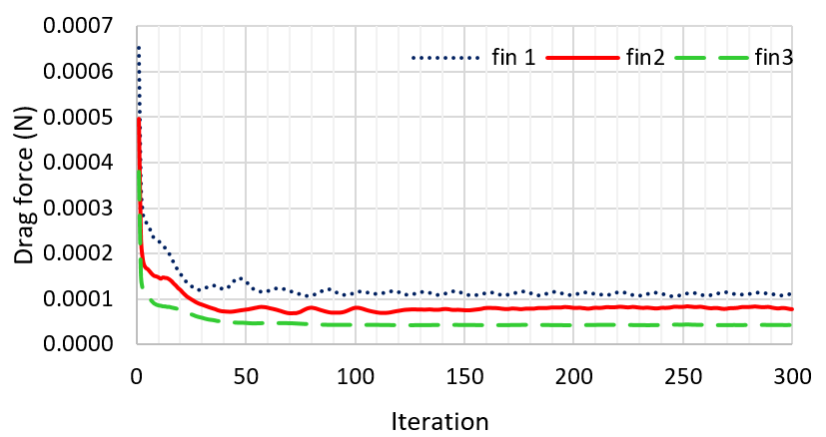

Fig.8: Drag force for each fin.

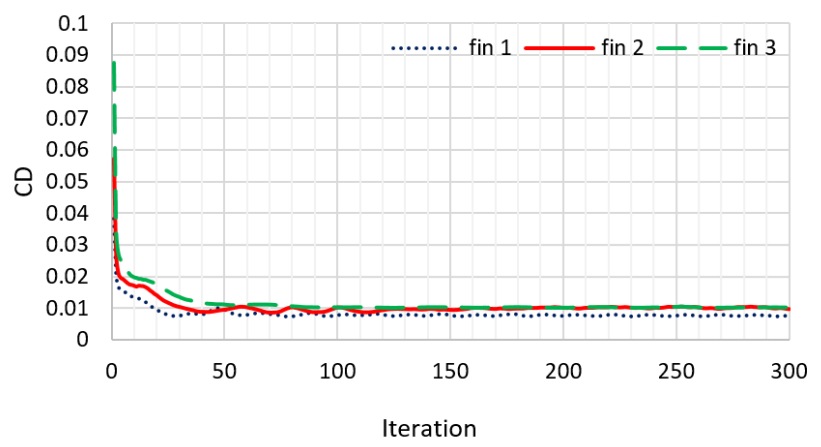

Fig.9: Drag coefficient for each fin. 


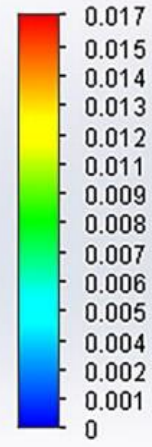

Velocity [m/s]

Velocity at power stroke: contours

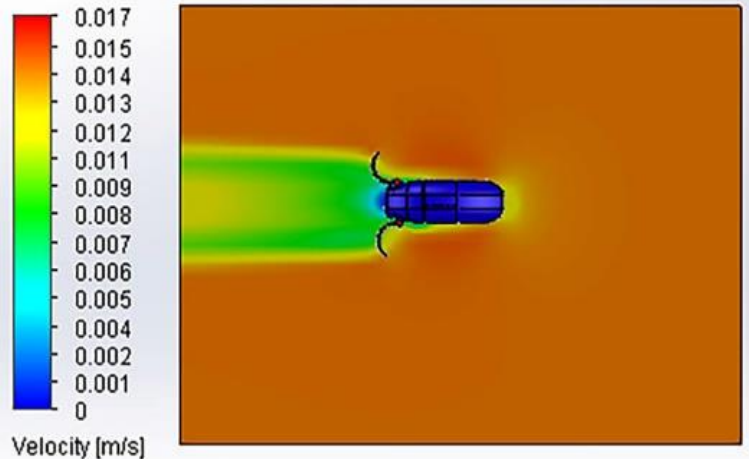

Velocity at recovery stroke: contours

Fig. 10: Flow contours during power and recovery strokes.
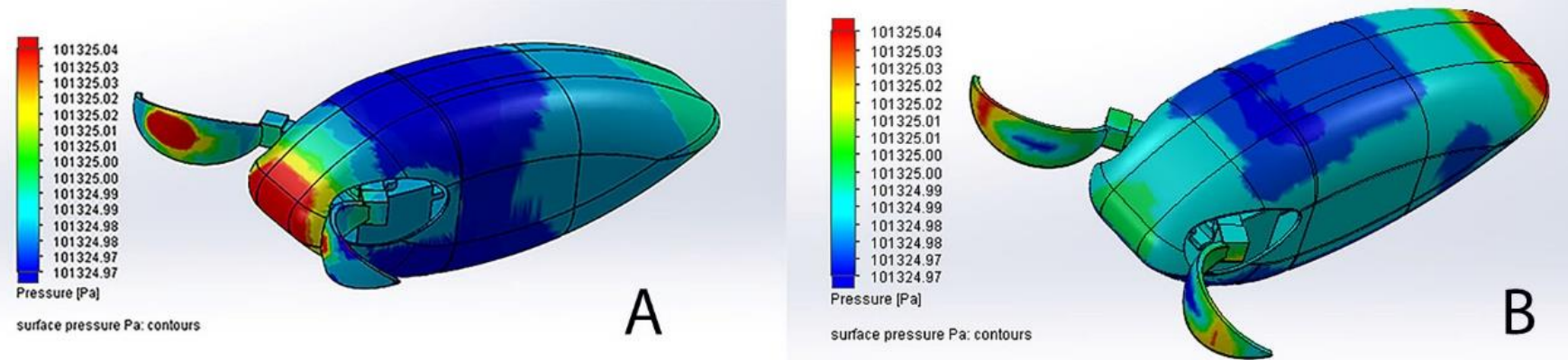

Fig.11: Pressure distribution on the robot's surface. A) Power stroke. B) Recover stroke.

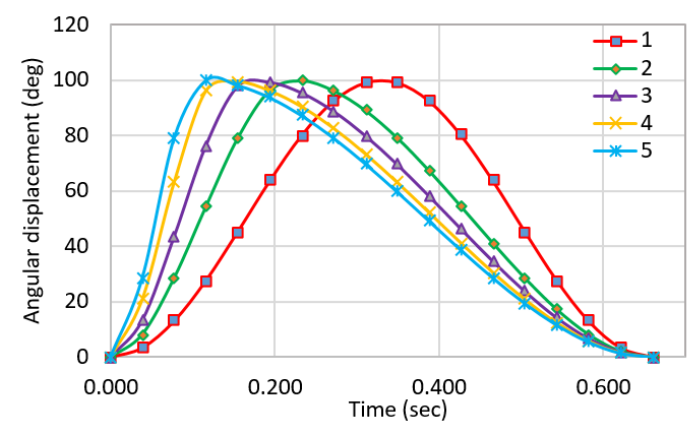

Motion commands are sent to the controller via the $\mathrm{HC}-06$ Bluetooth module, four $1.5 \mathrm{~V}$ AA batteries were used to supply the robot with the required energy as shown in Fig. 15 . Water density is assumed at $1000 \mathrm{~kg} / \mathrm{m} 3$. It is worthy to mention that all inner electronic devices are covered with NANO PROTECH coating technology spray to protect them from direct contact with water.

Fig.12: Input signals of proposed swimming robot.
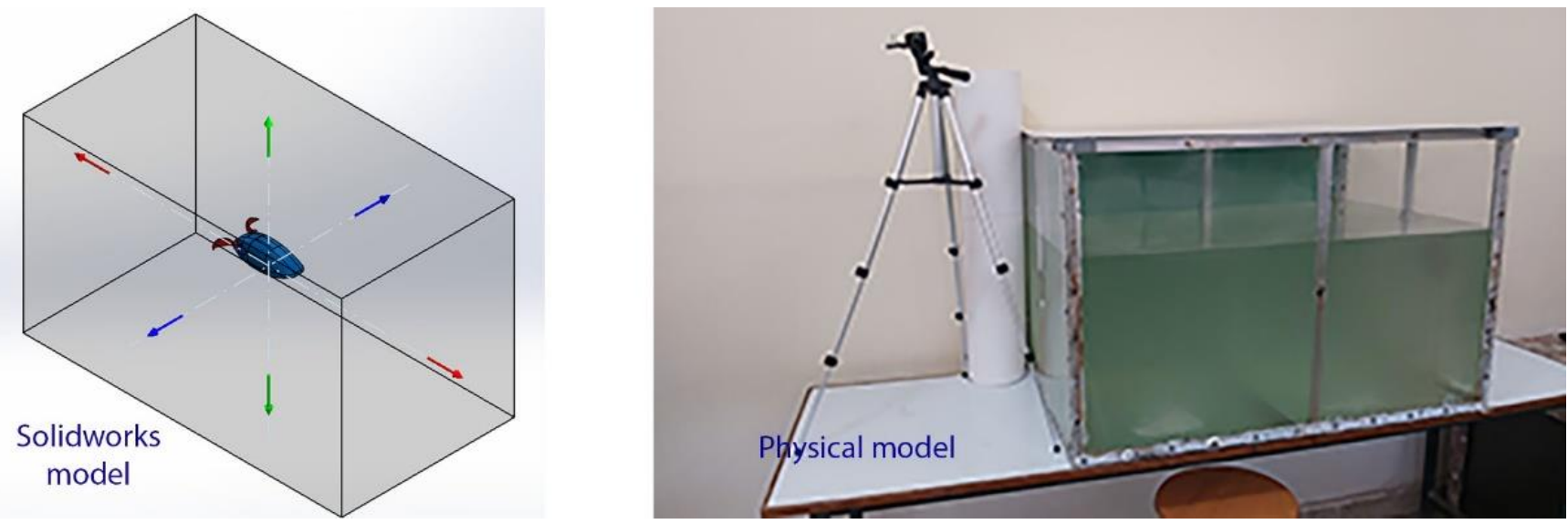

Fig.13: Swimming robot environment. 


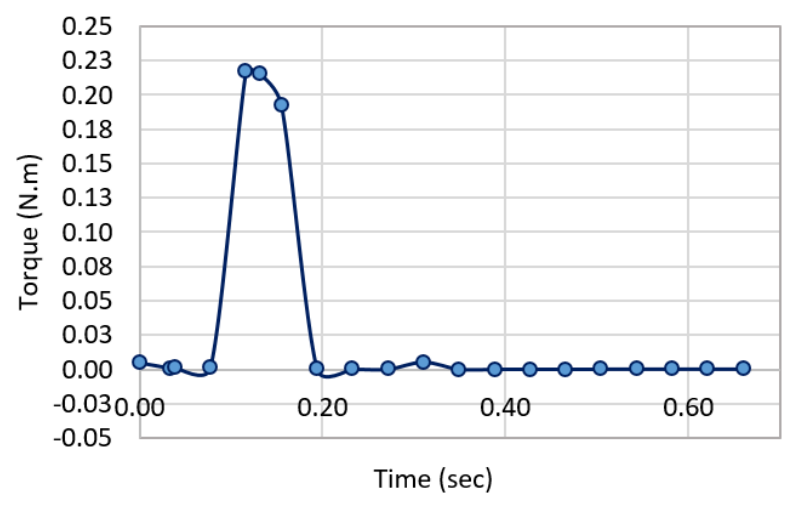

Fig. 14: Servomotor torque calculation.

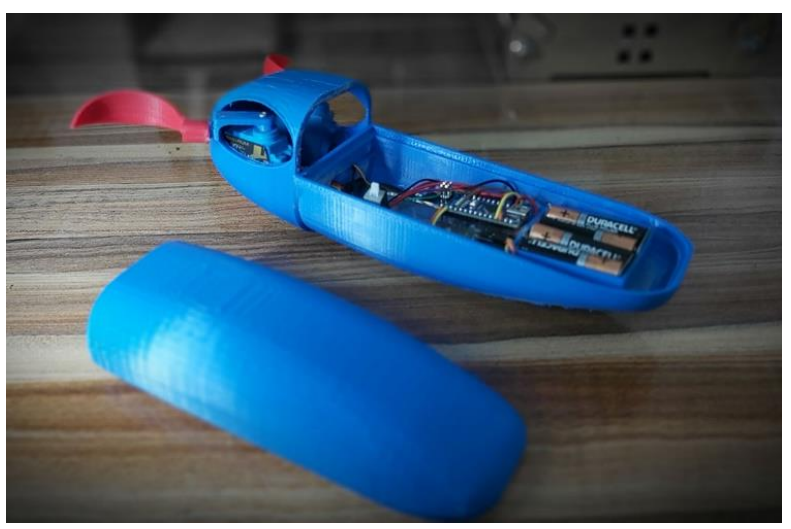

Fig. 15: Complete design of the proposed model.

Finally, for further validation of the above design, the efficiency has been calculated of our robot in terms of Strouhal number as shown in Fig. 16, which Strouhal number for biological fish is usually in the range of 0.05-0.6. From Fig. 16 can be noticed that it can get about 3.8 value of Strouhal number at 3:1 ratio. This value is much greater than that range one reason is that when physical fish swims, it does not depend purely on its pectoral fins as presented in this study rather it can use tail and other fins also. This result in a relative low swimming velocity and consequence high Strouhal numbers.

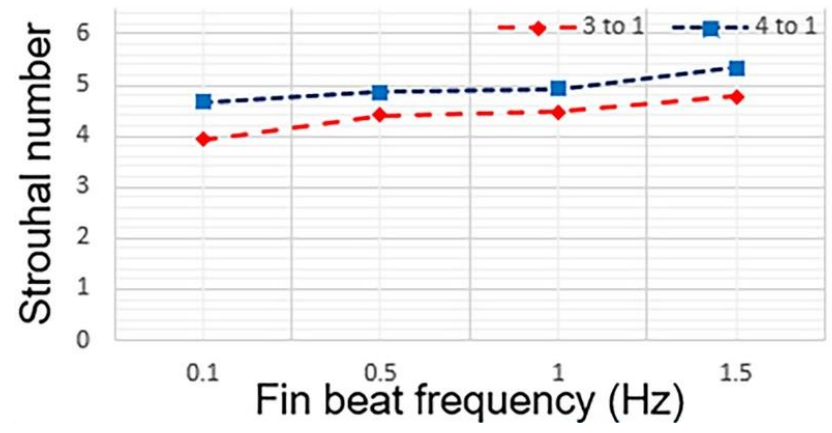

Fig.16: Strouhal number calculation.

\section{Conclusion}

This study represents the first step in designing a Labriform swimming robot with pectoral fins only, first, the effect of pectoral fins in producing the highest thrust while keeping the drag to a minimum has been studied. With the aid of computational fluid dynamics tools provided by solidworks ${ }^{\circledR}$, we have able to pick up the optimum shape and size from three concave pectoral fins, each one with a specific aspect ratio, the results showed that the lowest aspect ratio fin (fin1 in section II) produces the highest drag. Whereas the highest aspect ratio fin (fin3 in section 2) gives the lowest drag and thrust. However, an intermediate aspect ratio fin (fin 2 in section 2) will produce a valuable thrust and an accepted drag. Next, the complete design of the robot has been proposed, the complete design of the body and pectoral fins have been subjected to CFD analysis to show the validity of the proposed model. All rigid elements of the designed robot have been printed by 3D printer of PLA plastic material. The robot's body shape is designed with approximately an elliptical cross-sectional area. Finally, our analysis has been supported by calculating Strouhal number, although the obtained value is still large in comparison with the biological fish, but it still a good achievement in the design of the Labridae robot. Therefore, the results of these experiments prove the success of the proposed design and it can be used as a Labriform mode swimming robot.

\section{CONFLICT OF INTEREST}

The authors have no conflict of relevant interest to this article.

\section{REFERENCES}

[1] G. Karpouzian, G. Spedding, and H. K. Cheng, "Lunate-tail swimming propulsion. Part 2. Performance analysis," Journal of Fluid Mechanics, vol. 210, pp. 329-351, 1990.

[2] Y. M. Su, Q. M. Cao, and W. C. Lai, "Analysis of the hydrodynamics of a pectoral fin propulsor," Journal of Harbin Engineering University, vol. 28, no. 7, pp. 727-733, 2007.

[3] Y. J. Park, T. M. Huh, D. Park, and K. J. Cho, "Design of a variable-stiffness flapping mechanism for maximizing the thrust of a bio-inspired underwater robot," Bioinspiration \& Biomimetics, vol. 9, no. 3, Article ID 036002, 2014.

[4] M. Ebrahimi and M. Abbaspour, "A comparative numerical study on the performances and vortical patterns of two bioinspired oscillatory mechanisms: undulating and pure heaving," Applied Bionics and Biomechanics, vol. 2015, Article ID 325934, 25 pages, 2015.

[5] P. W. Webb, "The biology of fish swimming," in Mechanics and Physiology of Animal Swimming, Cambridge University Press, pp. 45-62, 1994.

[6] Ningyu $\mathrm{Li}$ and Yumin $\mathrm{Su}$, "Fluid Dynamics of Biomimetic Pectoral Fin Propulsion Using Immersed Boundary Method", Hindawi Publishing Corporation Applied Bionics and Biomechanics Volume 2016, 
Article ID 2721968, 22 pages http://dx.doi.org/10.1155/2016/2721968.

[7] P. E. Sitorus, Y. Nazaruddin, E. Leksono and A. Budiyono," Design and implementation of paired pectoral fins locomotion of labriform fish applied to a fish robot", science direct, journal of bionic engineering, vol. 6, pp. 37-45, 2009.

[8] A. Raj and A. Thakur," Fish-inspired robots: design, sensing, actuation, and autonomy - a review of research", bio inspiration and bio mimetic, April 19, 2016.

[9] N. Li and Y. Su," Fluid dynamics of biomimetic pectoral fin propulsion using immersed boundary method", applied bionics and biomechanics, vol. 2016, 22 pages, 2016.

[10] J. J. Videler, Fish Swimming. London: Chapman \& Hall, 1993.

[11] Farah Abbas Naser, Mofeed Rashid, "Design, modeling, and experimental validation of a concave-shape pectoral fin of labriform-mode swimming robot", Engineering Reports, Vol. 1, No. 5, pp. 1-17, 2019. doi: 10.1002/eng2.12082.

[12]R. W. Blake, "The swimming of the mandarin fish Synchropus picturatus (Callionyiidae: Teleostei)," J. Marine Biol. Assoc. U.K., vol. 59, pp. 421-428, 1979.

[13] S. Vogel, Life in moving fluids, Princeton: Princeton University Press, 1994.

[14]Eliot G. Drucker, Jeffrey A. Walker, Mark W. Westneat," Functional Biology of Pectoral Fin Swimming in Fishes", 2005. DOI: 10.1016/S1546-5098(05)23010-8.

[15]P. C. Wainwright, D. R. Bellwood, and M. W. Westneat, "Ecomorphology of locomotion in labrid fishes", Env. Biol. Fish, Vol. 65, pp. 47-62, 2002.

[16] J. A. Walker, and M. W. Westneat, "Performance limits of labriform propulsion and correlates with fin shape and motion", J. Exp. Biol., vol. 205, pp. 177-187.

[17] D. R. Bellwood, and P. C. Wainwright, "Locomotion in labrid fishes: implications for habitat use and cross-shelf biogeography on the Great Barrier Reef", Coral Reefs, vol. 20, pp. 139- 150, 2001.

[18] C. J. Fulton, D. R. Bellwood, and P. C. Wainwright, "The relationship between swimming ability and habitat use in wrasses (Labridae)", Mar. Biol., vol. 139, pp. 25-33, 2001.

[19] Combes, S. A. and Daniel, T. L. (2001). Shape, flapping and flexion: wing and fin design for forward flight. J. Exp. Biol. 204, 2073-2085.

[20] J. A. Walker, and M. W. Westneat, "Labriform propulsion in fishes: kinematics of flapping aquatic flight in the bird wrasse Gomphosus varius (Labridae)", J. Exp. Biol., vol. 200, pp. 1549-1569, 1997.

[21]C. M. Breder, "The locomotion of fishes", Zoologica (N.Y.), vol. 4, pp. 159-297, 1926.

[22] R. W. Blake, “On ostraciiform locomotion', J. Mar. Biol. Ass. U.K., vol. 57, pp. 1047-1055, 1977.

[23] C.C. Lindsey, "Form, function and locomotory habits in fish," in Fish Physiology, vol. VII Locomotion, edited by W.S. Hoar and D.J. Randall. New York: Academic Press, pp. 1- 100, 1978.
[24] Michael Sfakiotakis, David M. Lane and John Bruce Davies, "Review of Fish Swimming Modes for Aquatic Locomotion", IEEE Journal of Oceanic Engineering, vol. 24, no. 2, pp. 237 - 252, 1999. DOI:10.1109/48.757275.

[25] Farah Abbas Naser, Mofeed Rashid," Effect of Reynold Number and Angle of Attack on the Hydrodynamic Forces Generated from A Bionic Concave Pectoral Fins IOP Conference Series: Materials Science and Engineering, vol. 745, 2019.

[26] S. B. Behbahani and X. Tan, "Role of pectoral fin flexibility in robotic fish performance," J. Nonlinear Sci., vol. 27, no. 4, pp. 1155-1181, 2017.

[27] S. B. Behbahani and X. Tan, "Bio-inspired flexible joints with passive feathering for robotic fish pectoral fins," Bioinspiration \& Biomimetics, vol. 11, no. 3, 036009, 2016.

[28]C. Eloy, "Optimal Strouhal number for swimming animals”, J. Fluids Struct., vol. 30, pp. 205-218, 2012.

[29] Aureli M, Kopman V and Porfiri M, "Free-locomotion of underwater vehicles actuated by ionic polymer metal composites", IEEE/ASME Trans. Mechatronics, vol. 15, pp. 603-614, 2010.

[30] Quinn D B, Lauder G V and Smits A J, "Maximizing the efficiency of a flexible propulsor using experimental optimization", J. Fluid Mech., pp. 430-48, 2015.

[31]H. N. Arafat, D. J. Stilwell, and W. L. Neu, "Development of a dynamic model of a small high-speed autonomous underwater vehicle," in Proc. Oceans 2006, Boston, MA, pp. 1-6.

[32]T. I. Fossen, "Guidance and Control of Ocean Vehicles", 1st ed. New York: Wiley, 1994.

[33] M. T. Rashid and A. T. Rashid, "Design and implementation of swimming robot based on labriform model", Al-Sadeq Int. Conf. on Multidisciplinary in IT and Commun. Sci. and App. (AIC-MITCSA), Iraq, 2016. 